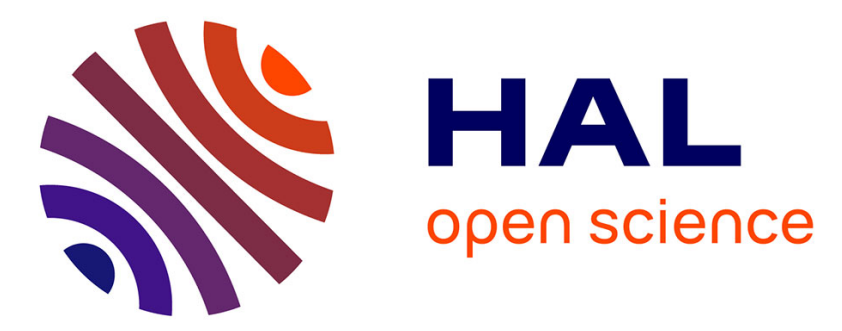

\title{
Modeling the impact of node speed on the ranging estimation with UWB body area networks
}

\author{
Arturo Guizar, Claire Goursaud, Jean-Marie Gorce
}

\section{To cite this version:}

Arturo Guizar, Claire Goursaud, Jean-Marie Gorce. Modeling the impact of node speed on the ranging estimation with UWB body area networks. IEEE International Symposium on Personal, Indoor and Mobile Radio Communication (PIMRC), Aug 2015, Hong Kong, China. 10.1109/PIMRC.2015.7343490 . hal-01246926

\section{HAL Id: hal-01246926 \\ https://hal.science/hal-01246926}

Submitted on 20 Dec 2015

HAL is a multi-disciplinary open access archive for the deposit and dissemination of scientific research documents, whether they are published or not. The documents may come from teaching and research institutions in France or abroad, or from public or private research centers.
L'archive ouverte pluridisciplinaire $\mathbf{H A L}$, est destinée au dépôt et à la diffusion de documents scientifiques de niveau recherche, publiés ou non, émanant des établissements d'enseignement et de recherche français ou étrangers, des laboratoires publics ou privés. 


\title{
Modeling the Impact of node speed on the ranging estimation with UWB Body Area Networks
}

\author{
Arturo Guizar, Claire Goursaud and Jean-Marie Gorce \\ University of Lyon, INRIA \\ INSA Lyon, CITI-INRIA, F-69621, Lyon, France \\ \{arturo.guizar, claire.goursaud, jean-marie.gorce\}@insa-lyon.fr
}

\begin{abstract}
The purpose of this paper is to evaluate the impact of the node speed on the ranging estimation for location applications with Wireless Body Area Networks (WBAN). While estimated with the 3-Way ranging protocol (3-WR), this distance between two nodes placed on the body can be affected by the human movements. Thus, we study theoretically the ranging error with the 3-WR, based on a perfect channel, a MAC layer based on TDMA using two scheduling strategies (Single node localization (P2P-B) and Aggregated \& Broadcast (A\&B)) and a PHY layer based on Ultra Wideband (IR-UWB). We demonstrate the accuracy of the model, and show that the distance error is highly correlated with the speed of nodes, while the associated mobility model has an impact on the design of MAC strategies by simulation.
\end{abstract}

\section{INTRODUCTION}

Wireless Body Area Networks (WBAN) refers to a new disruptive technology composed of a group of nodes embedded on a person. These nodes communicate with each other for diverse applications, e.g. sport analysis, civil security or ehealth [1]. In this context, radio-location techniques are highly studied, because there is a strong interest for the analysis of body movement. This is possible, among others techniques, with the transmission of pulses in Ultra Wideband (IR-UWB) [2]. The high temporal resolution of the pulses makes possible to calculate the distance between two nodes by estimating the the Time of Flight (ToF) of three packets as defined by the Three Way Ranging Protocol (3-WR). Several research works have addressed the localization problems with IR-UWB, particularly to compensate the clock's desynchronization and the channel effects [3]. However, most of studies suggest solutions for static or low mobile nodes in Wireless Sensor Networks (WSN). In the case of WBAN, nodes are affected by different variations in the speed of the human body. Therefore, the distances estimated vary during the 3-WR transactions, inducing positioning errors (Fig. 1).

The goal of this work is to analyze the impact of speed on the ranging estimation. For this, we propose a theoretical model that takes into account this impact. Then, we show the relation of speed of nodes with the estimated distances by evaluating our theoretical model with the simulated ranging estimation. Moreover, we give some important key points to consider when designing MAC protocols to perform localization applications. Our results show a high correlation between the type of performed movement (and therefore, the speed of nodes) and the error in the ranging estimation.

The remaining of the paper is organized as follows. Section II briefly describes the network configuration involved for the localization purposes, along with the realistic mobility model description. In Section III, we present our theoretical model and we describe the MAC strategies used for the simulation by giving the main assessments. Section IV reports the results for both theoretical and simulated ranging errors obtained through discrete-event simulation. Finally, Section V draws the conclusion and perspectives of this work.

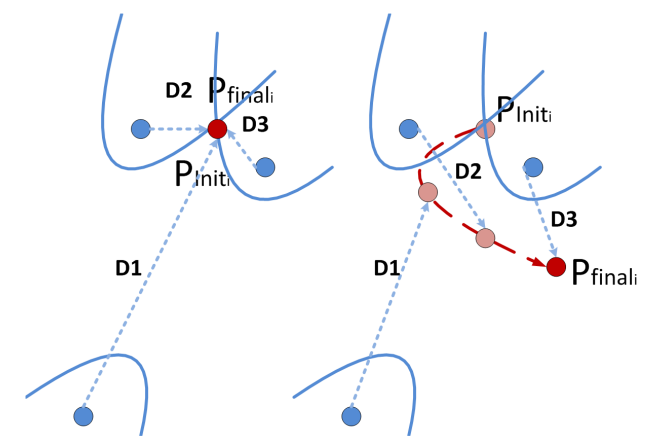

Fig. 1. Ranging error estimation between static node and mobile node

\section{System MODEL}

\section{A. Network Topology}

In this work, we consider a wireless body area network (WBAN) embedded on a person. This network is considered as full-mesh and all nodes $\left(N_{t}\right)$ can directly communicate in pairs. We use a physical layer based on Ultra Wide Band (UWB) pulses. The network is characterized by two types of sensors: the anchor nodes that have perfect knowledge of their position at any time and the mobile nodes who want to estimate their position. The anchors perform a Local Coordinate System (LCS) associated with the body, which is mobile under a Global Coordinate System (GCS). To achieve the localization, a node $i$ must estimate its distance with an anchor $j$, denoted $\hat{d}_{i j}(t)$, then estimate its position $\hat{P}_{i}(t)$.

\section{B. Ranging estimation with $3 W R$}

As shown in Figure 2, the distance between an anchor node and a mobile node can be evaluated with the Time of Arrival (ToA) estimation of 3 packets sent at different instants, as defined by the 3-Way Ranging protocol (3-WR) [2], i.e. the 3WR is an extension of the 2-Way Ranging protocol considering the drift clock between the nodes:

- $\quad$ At time $t_{0}$, the node $i$ send a Request packet $\left(Q_{i j}\right)$ to the anchor $j$. 
- $\quad$ At time $t_{1}$, the anchor $j$ answers with a Response 1 packet $\left(R 1_{i j}\right)$. Thus, the node $i$ is able to estimate the Time of Flight (ToF) of the pulses and therefore, a first ranging estimation (2-WR).

- $\quad$ Finally, at $t_{2}$, the anchor $j$ sends a Response 2 packet $\left(R 2_{i j}\right)$. In fact, since the localization precision can be reduced by non-synchronization of clocks between the nodes, this last packet allows to evaluate and compensate the clock drift.

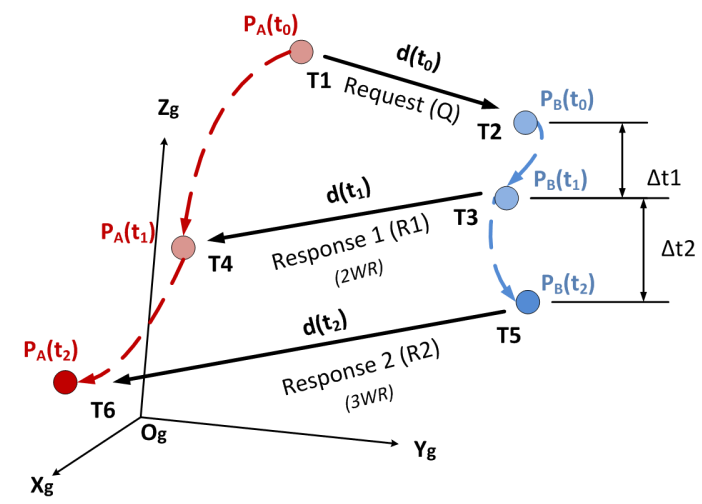

Fig. 2. Three Way Ranging protocol applied with mobile nodes

From these transactions, we define the times $T 1, T 3$ and $T 5$ as the moments where the nodes sends the $Q_{i j}, R 1_{i j}$ and $R 2_{i j}$ packets respectively. Similarly, we note $T 2, T 4$ and $T 6$ as the ToA of these same packets respectively. Thus, the distance $\hat{d}_{i j}(t)$ is evaluated with the Equation (1):

$$
\hat{d}_{i j}(t)=\frac{1}{2} c\left[\left(\left(T_{4}-T_{1}\right)-\Delta t 1\right)-\left(\left(T_{6}-T_{4}\right)-\Delta t 2\right)\right]
$$

where $c$ represents the speed of pulses transmission (i.e. $c=$ $\left.3 * 10^{8} \mathrm{~m} / \mathrm{s}\right)$ and $\Delta t 1$ (resp. $\left.\Delta t 2\right)$ is the delay between $\left(T_{3}-T_{2}\right)$ (resp. $\left(T_{5}-T_{3}\right)$ ).

\section{Mobility Model}

In this study, we consider a realistic Yoga Model, obtained by measurement. The mobility traces were obtained during the measurement campaign related to the CORMORAN project at the M2S laboratory, ENS Cachan, France in June 2014 [4]. This model was generated using a motion capture system (Vicon [5]) based on infrared at a rate of $100 \mathrm{~Hz}$. For this, we deployed 16 cameras in a confined space of $10 \times 6 \mathrm{~m}^{2}$. Thus, we were able to calculate the position and the instantaneous velocity of each node.

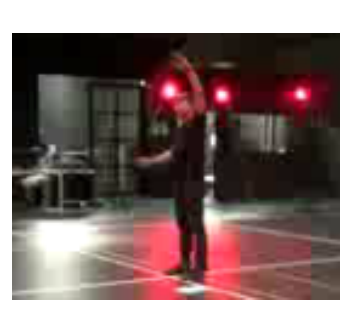

(a)

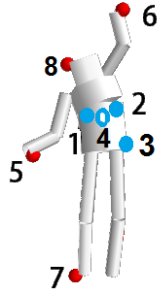

(b)
Fig. 3. (a) Camera snapshot during the measurement of the Yoga activity. (b) Multi-cylinder Body reconstruction - the red (resp. blue) points refer to the mobile nodes (resp. anchors).
Accordingly, we consider a scenario of $100 \mathrm{~s}$ which consist in the representation of Yoga activity. The interest in this scenario is the study of the presence or absence of movement which separates the impact of the speed and the other error factors on the accuracy. The series of the static positions (realizing yoga postures (e.g. put both feet together and hands above the head)) is performed in the same place, so only the articulations of the body perform a movement. Here, we consider a network composed of 4 anchors and 4 mobile nodes, as shown in Figure 3(b). The anchors are positioned on the most static parts of the body: the right chest $\left(A_{1}\right)$, the left chest $\left(A_{2}\right)$, the left hip $\left(A_{3}\right)$ and the back $\left(A_{4}\right)$. The mobile nodes are located on the right arm $\left(N_{5}\right)$, the left arm $\left(N_{6}\right)$, the right foot $\left(N_{7}\right)$ and the head $\left(N_{8}\right)$.

\section{MODELING THE RANGING ESTIMATION WITH NODES UNDER MOBILITY}

As presented in Section II-B, the 3-Way Ranging protocol was proposed for the ranging estimation between quasi-static nodes. However, WBANs are exposed to strong mobility, having a direct impact on the estimated distances $\hat{d}_{i j}(t)$. In fact, the 3-WR packets travel through varying distances, hence, the ToF change as well. In our previous work [6], we showed that the ranging accuracy depends on the duration of $\Delta t 1$ and $\Delta t 2$. In particular, the delay $\Delta t 1$ to send the first response $R 1_{i j}(t)$ has more impact than the time taken $\Delta t 2$ for the second response $R 2_{i j}(t)$. Therefore, the nodes present a bigger displacement when these delays increase.

\section{A. Theoretical Model}

In this study, we aim to quantify the impact of node speed on the ranging estimation. For this, we consider a three-Dimensional Euclidean space $\left(O_{g}, x_{g}, y_{g}, z_{g}\right)$. We define $\vec{P}(t)=\overrightarrow{O g P}$ as the position vector between the origin point and the node's position at time $t$. Moreover, we note $\vec{d}_{i j}(t)=\vec{P}_{j}(t)-\vec{P}_{i}(t)$ as the distance vector between node $i$ and anchor $j$ during a 3 -WR packet transmission $\left(Q_{i j}, R 1_{i j}\right.$ and $R 2_{i j}$ ) at a given moment $(\mathrm{t})$.

However, the 3-WR packets (Fig. 2) are sent at different moments, which are long enough to let the nodes move. Therefore, the initial distance is affected by different displacements $\left(t_{0} \Rightarrow t_{1}\right.$ and $\left.t_{1} \Rightarrow t_{2}\right)$, leading to 3 distances for the same ranging estimation. Note that the distance vector norm is expressed in terms of the speed of propagation of an UWB pulse as $\left\|\vec{d}_{i j}(t)\right\|=c *\left(T_{R j}-T_{E i}\right)$, where $T_{E i}$ (resp. $T_{R j}$ ) represents the moment of a packet transmission (resp. reception) by the node $i$ (resp. $j$ ) at time $t$. As result, Equation (1) can be written as:

$$
\begin{aligned}
\hat{d}_{i j}(t)= & \frac{1}{2} c\left[\left(\left(T_{4}-T_{3}\right)+\left(T_{2}-T_{1}\right)\right)-\left(\left(T_{6}-T_{5}\right)-\left(T_{4}-T_{3}\right)\right)\right] \\
\hat{d}_{i j}(t)= & \frac{1}{2}\left[\left(\left\|\vec{d}_{i j}\left(t_{1}\right)\right\|+\left\|\vec{d}_{i j}\left(t_{0}\right)\right\|\right)-\left(\left\|\vec{d}_{i j}\left(t_{2}\right)\right\|-\left\|\vec{d}_{i j}\left(t_{1}\right)\right\|\right)\right] \\
& \hat{d}_{i j}(t)=\frac{1}{2}\left[\left\|\vec{d}_{i j}\left(t_{0}\right)\right\|+2\left\|\vec{d}_{i j}\left(t_{1}\right)\right\|-\left\|\vec{d}_{i j}\left(t_{2}\right)\right\|\right]
\end{aligned}
$$

In our model, we aim to express $\hat{d}_{i j}(t)$ as a function of the node speed and the initial distance $\vec{d}_{i j}\left(t_{0}\right)=\vec{P}_{j}\left(t_{0}\right)-\vec{P}_{i}\left(t_{0}\right)$. For this, we define the average speed of the node $i$ at time 

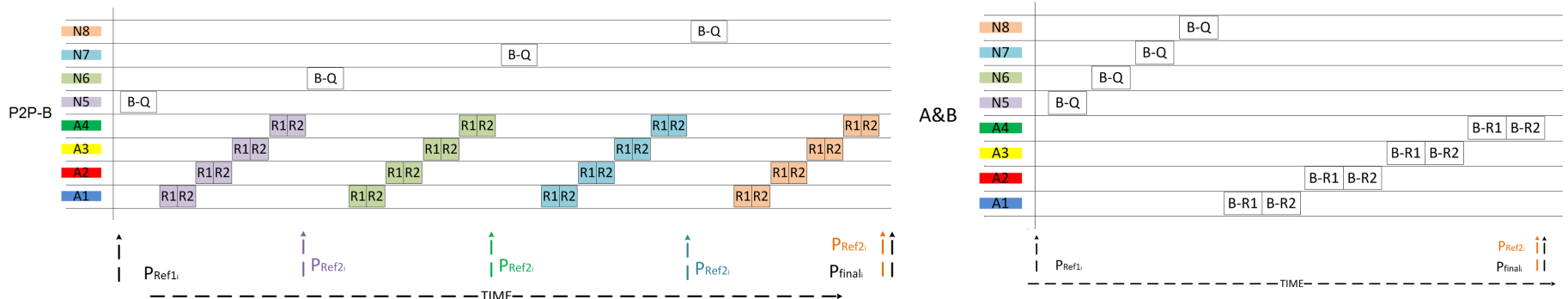

Fig. 4. Details of the P2P-B and A\&B scheduling

$t$ as $\vec{V}_{i}(t)=\frac{\vec{P}_{i}(t)-\vec{P}_{i}(t-1)}{\delta t}$. Accordingly, the distance vector $\vec{d}_{i j}\left(t_{1}\right)$, after the first movement $t_{0} \Rightarrow t_{1}$, can be expressed with the Chasles relation $(\overrightarrow{A D}=\overrightarrow{A B}+\overrightarrow{B C}-\overrightarrow{D C})$ as:

$$
\begin{aligned}
\left(\vec{P}_{j}\left(t_{1}\right)-\vec{P}_{i}\left(t_{1}\right)\right)= & \left(\vec{P}_{j}\left(t_{0}\right)-\vec{P}_{i}\left(t_{0}\right)\right)-\left(\vec{P}_{i}\left(t_{1}\right)-\vec{P}_{i}\left(t_{0}\right)\right) \\
& +\left(\vec{P}_{j}\left(t_{1}\right)-\vec{P}_{j}\left(t_{0}\right)\right) \\
\vec{d}_{i j}\left(t_{1}\right)= & \vec{d}_{i j}\left(t_{0}\right)-\vec{V}_{i}\left(t_{1}\right)\left(T_{4}-T_{1}\right)+\vec{V}_{j}\left(t_{1}\right) \Delta t 1
\end{aligned}
$$

When using the same relation for $\vec{d}_{i j}\left(t_{2}\right)$, we can obtain the following system of equations with 3 variables:

$$
\left\{\begin{array}{l}
\vec{d}_{i j}\left(t_{0}\right)=\vec{P}_{j}\left(t_{0}\right)-\vec{P}_{i}\left(t_{0}\right) \\
\vec{d}_{i j}\left(t_{1}\right)=\vec{d}_{i j}\left(t_{0}\right)-\vec{V}_{i}\left(t_{1}\right)\left(T_{4}-T_{1}\right)+\vec{V}_{j}\left(t_{1}\right) \Delta t 1 \\
\vec{d}_{i j}\left(t_{2}\right)=\vec{d}_{i j}\left(t_{1}\right)-\vec{V}_{i}\left(t_{2}\right)\left(T_{6}-T_{4}\right)+\vec{V}_{j}\left(t_{2}\right) \Delta t 2
\end{array}\right.
$$

This proposition is strictly true if we assume that nodes do not move during 1 packet transmission. In practice, this assumption can be considered if we neglect the speed of a node $(<10 \mathrm{~m} / \mathrm{s})$ compared to the pulse speed propagation $(c)$. Therefore, the movement of nodes between $\left(T_{4}-T_{3}\right)$ and $\left(T_{2}-T_{1}\right)$ are negligible. As result, $T_{4}-T_{1}$ can be written as $T_{4}-T_{1}=\left(T_{4}-T_{3}\right)+T_{3}-T_{2}+\left(T_{2}-T_{1}\right) \simeq \Delta t 1$. Similarly, $T_{6}-T_{4} \simeq \Delta t 2$ which yields to the simple formulation of the system of equations (3) as:

$$
\left\{\begin{array}{l}
\vec{d}_{i j}\left(t_{0}\right)=\vec{P}_{j}\left(t_{0}\right)-\vec{P}_{i}\left(t_{0}\right) \\
\vec{d}_{i j}\left(t_{1}\right)=\vec{d}_{i j}\left(t_{0}\right)-\left(\vec{V}_{i}\left(t_{1}\right)-\vec{V}_{j}\left(t_{1}\right)\right) \Delta t 1 \\
\vec{d}_{i j}\left(t_{2}\right)=\vec{d}_{i j}\left(t_{1}\right)-\left(\vec{V}_{i}\left(t_{2}\right)-\vec{V}_{j}\left(t_{2}\right)\right) \Delta t 2
\end{array}\right.
$$

\section{B. Scheduling MAC Protocols}

In this paper, we want to characterize only the impact of speed on the ranging estimation accuracy. For this, we assume a perfect channel and we ignore the shadowing effects of the body. Thus, for positioning in a three-dimensional space, the node estimates its distance with (at least) 4 anchors, and then calculates its position with a positioning algorithm (e.g. TDOA) [2]. The frame holds enough slots for an individual location of all nodes. Moreover, $\Delta t 1$ and $\Delta t 2$ are fixed by the scheduling strategies at the MAC layer. In order to validate our theoretical analysis, we implement two scheduling MAC strategies as the one described in [7]:

- Single node localization (P2P-B) where each node $i$ intend to send the requests $Q_{i}$ to the anchors in broadcast. Then, each anchor answers with $R 1_{j i}$ and $R 2_{j i}$ successively in single-links to the nodes.
- $\quad$ Aggregated and Broadcast $(\mathbf{A} \& \mathbf{B})$ [8] where nodes send the requests $Q_{i}$ in broadcast. Thereafter, each anchor $j$ gathers the ToA of each request and sends an aggregated response $\left(R 1_{j}\right)$ to all nodes, followed by the second response $\left(R 2_{j}\right)$. Thus, A\&B increases the $\Delta t 1$ delay even though it reduces the frame size.

\section{PERFORMANCE EVALUATION}

\section{A. Simulation tools and methodology}

In this work, we adopt a discrete event simulation with WSNet [9], suitable for the test of our theoretical model on the experiment movement traces.In particular, we implemented an UWB PHY layer as defined by the standard IEEE802.15.6 in default mode (OOK modulation and $0.4875 \mathrm{Mb} / \mathrm{s}$ ). At the MAC layer, we implemented a protocol based on TDMA, the P2P-B and A\&B algorithms, as detailed in Section III-B. Finally, we created a mobility model which exploits the traces of both scenarios acquired during the CORMORAN project, as described in Section II-C.

Within this framework, we run the simulation on the $100 \mathrm{~s}$ of data acquisition with our mobility scenario and then, we compare with the calculated theoretical distance with the node speed and the delays $\Delta t 1$ and $\Delta t 2$ (depending on the MAC scheduling employed for each simulation). The temporal data resolution is the one from the Vicon cameras calibration (one position measured every $10 \mathrm{~ms}$ ).

\section{B. Relation between nodes speed and ranging estimation}

First, we consider the Yoga scenario in order to evaluate our model in a case with a controlled and slow evolution of nodes positions. Figure 5(a) shows the speed of nodes calculated from the mobility model. The anchors are quasi-static (Figure not presented), contrary to the mobile nodes (Fig. 5(a)) which move according to the performed Yoga positions $(<3 \mathrm{~m} / \mathrm{s})$. Therefore, we can assume that our ranging estimation will be affected mostly by the mobile nodes movement.

For a preliminary analysis, we evaluate by simulation the error on the estimated distances and quantify the impact on the design of the MAC layer. For this purpose, we simulated the $\mathrm{P} 2 \mathrm{P}-\mathrm{B}$ and $\mathrm{A} \& \mathrm{~B}$ strategies to perform the $3-\mathrm{WR}$ ranging estimation for the Yoga scenario. For our network composed of 4 anchors and 4 nodes, we use a TDMA frame duration of $\approx$ $54 \mathrm{~ms}$ for $\mathrm{P} 2 \mathrm{P}-\mathrm{B}$ and $\approx 18 \mathrm{~ms}$ for A\&B. Figures $5(\mathrm{~b})$ and $5(\mathrm{c})$ represents the ranging error with $\mathrm{P} 2 \mathrm{P}-\mathrm{B}$ and $\mathrm{A} \& \mathrm{~B}$ respectively, between the mobile nodes and the anchor $N_{4}$ (which is the worst case because of a bigger $\Delta t 1$ ). 


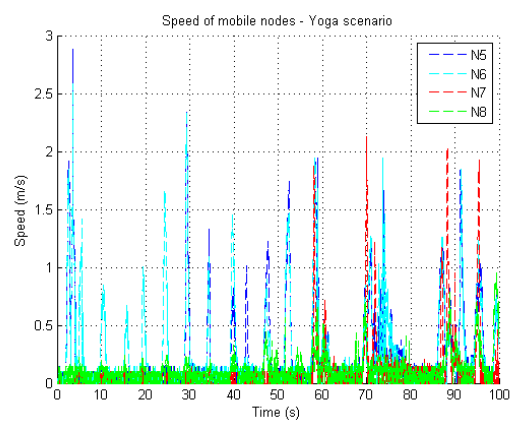

(a) Speed of mobile nodes.

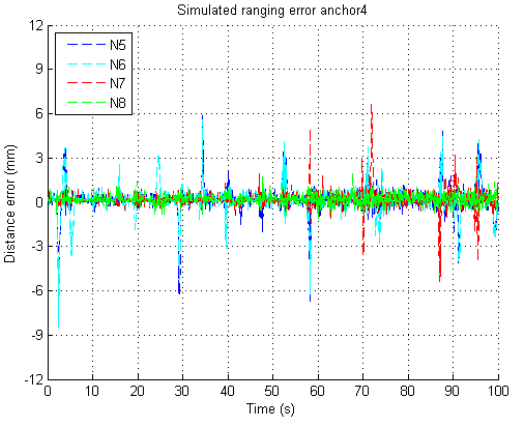

(b) Simulated ranging error with P2P-B.

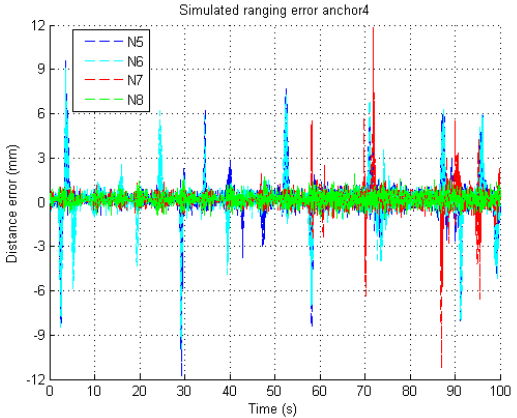

(c) Simulated ranging error with A\&B.

Fig. 5. Calculated speed of nodes and simulated ranging errors between mobile nodes and anchor $A_{4}$ with the Yoga scenario.

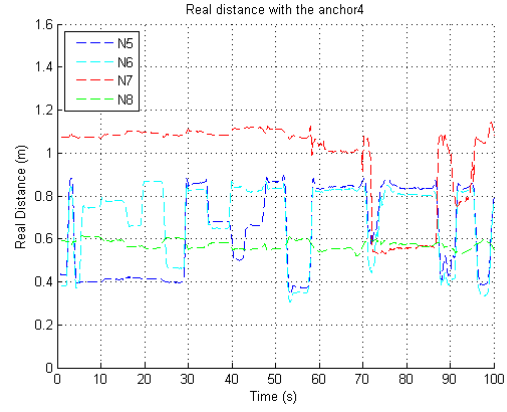

(a) Real distance between nodes and $A_{4}$.

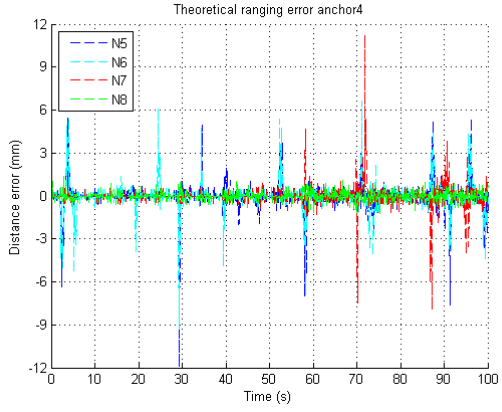

(b) Theoretical ranging error with P2P-B delays.

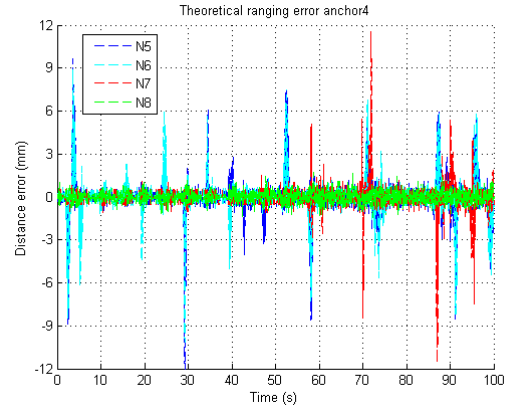

(c) Theoretical ranging error with A\&B delays.

Fig. 6. Validation of the theoretical ranging errors between mobile nodes and anchor $A_{4}$ with the Yoga scenario.

First, we can observe that the ranging error (for both strategies) follows the evolution of the node speed (Fig. 5(a)). Accordingly, we can easily distinguish the static periods (where all nodes have a low speed leading to small errors), and the activity periods (in which at least one node is moving). In fact, we note that not all the nodes move simultaneously, only the hands $\left(N_{5}\right.$ and $\left.N_{6}\right)$ are always active. Thus, we can differentiate in Figure 6(b) the three main periods of activity: $\mathrm{Sb} 1(0-50 \mathrm{~s}), \mathrm{Sb} 2(50-70 \mathrm{~s})$ and Sb3 $(70-100 \mathrm{~s})$.

In the case of Sb1, the subject raised and dropped both hands several times above his head. Accordingly, the nodes on the hands show the most of variation errors for a node speed depending on the performed postures (Half moon and Mountain poses); whereas the nodes on the foot $\left(N_{7}\right)$ and the head $\left(N_{8}\right)$ are quasi-static. For Sb2, the person stretches his legs (Warrior pose), which yields in an error on the estimation with $N_{7}$.We can notice that, at the end of this phase, the foot node approaches to the anchor 4 (Tree pose), leading to a positive error. Finally, for $\mathrm{Sb} 3$, the subject leans forward to touch his feet with his hands (bend forward pose), which explains the error for nodes $N_{5}, N_{6}$ and $N_{7}$. Here, the mobile nodes come close to the anchors.

\section{Validation of the theoretical model}

In this second study, we aim to evaluate our theoretical model by quantifying the error produced by the node speed between the initial distance of a 3 -WR transaction $\left(\left\|\vec{d}_{i j}\left(t_{0}\right)\right\|\right)$ and the theoretical estimated distance $\left(\hat{d}_{i j}(t)\right.$, Eq. 2). Then, we compare this theoretical error with the error obtained by simulation (Figures 5 and 6). For this, we fixed $\Delta t 1$ and $\Delta t 2$ to the delays related with P2P-B and A\&B. Figure 6(b) (resp. $6(\mathrm{c})$ ) illustrates the theoretical ranging error on the distances between the nodes and the anchor $N_{4}$ with P2P-B (resp. A\&B). As before, the theoretical error is correlated with the evolution of the speed of mobile nodes (Fig. 5(a)).

If we analyze our theoretical errors (Figs. 6(b) and 6(c)) with the real distance between nodes and $A_{4}$ (Fig.6(a)), we can observe a clear correspondence with the variation of the distances estimated. In fact, when nodes and anchors move away (resp. get closer), there is an increase (resp. decrease) of the distance estimated by simulation, but also a theoretical negative (resp. positive) error. This is most visible with $N_{7}$ in Sb3 where we clearly observe that its distance with $A_{4}$ change from $\approx 1.1 \mathrm{~m}(70 \mathrm{~s})$ to $\approx 0.55 \mathrm{~m}(72 \mathrm{~s})$ leading into a positive error of $(\approx 1.2 \mathrm{~cm})$. This kind of information is important to collect in order to make a control of the distance estimations. Knowing when the error is positive or negative give us the key to infer when the nodes are getting closer or not.

Moreover, the theoretical results are similar to the simulated errors for both strategies. However, the error with A\&B has visibly an increased level of error in the ranging estimation compared to $\mathrm{P} 2 \mathrm{P}-\mathrm{B}$, yet this is not entirely true. In fact, the delay $\Delta t 1$ is shorter with P2P-B which favors the ranging accuracy (Section III). On the other hand, A\&B reduces the frame duration which means a greater detail of the estimated distances. This is due to the fact that $\mathrm{A} \& \mathrm{~B}$ performs 3 times more the motion capture than P2P-B. In other words, P2P-B is able to estimate accurate distances but makes a subsample 


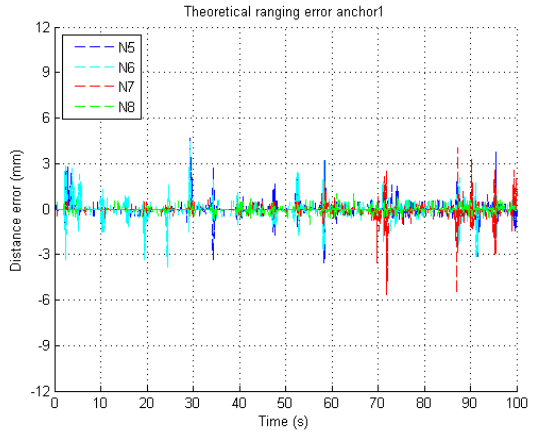

(a) Theoretical ranging error with $A_{1}$

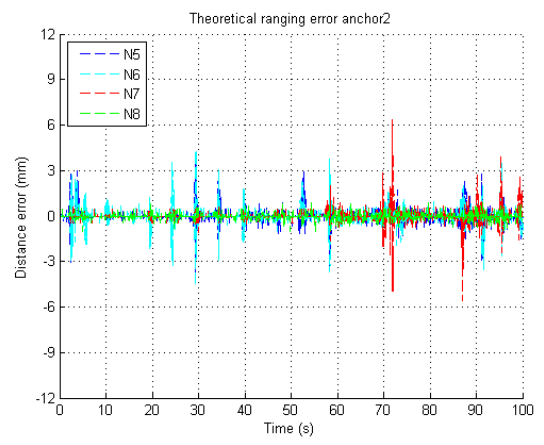

(b) Theoretical ranging error with $A_{2}$

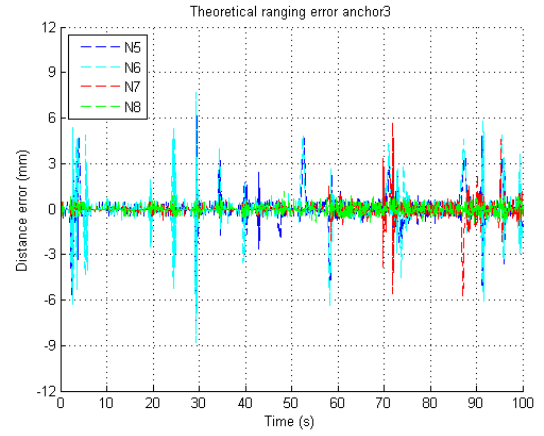

(c) Theoretical ranging error with $A_{3}$

Fig. 7. Ranging Error for the Yoga Scenario with the P2P-B MAC scheduling.

of motion, preventing the detection of some movements and consequently a greater temporal error, contrary to A\&B which detects the movements with more detail even though it presents a higher spatial error. To illustrate this last hypothesis, we can observe at second 30, a high peak variation of speed for $N_{5}$, which is not perceived by the P2P-B protocol, but it is detected by the A\&B protocol. This last observation is important because if a localization system requires precise detection of movements at a given time, P2P-B would not be the best option, since the duration of the frame skips some movements.

Finally, the difference between the simulated errors and theoretical errors (for both strategies) is due to the limit of simulation related to the mobility model. In our case, the vicon system was calibrated to detect the node's positions every $10 \mathrm{~ms}$. Since each MAC strategy has different frame duration, the ranging estimation will be affected by the mobility model sampling. However, the theoretical model has not such a problem because it calculates the speed progressively. To illustrate this, Figure 7 shows the theoretical error with the P2P-B delays for anchors $A_{1}, A_{2}$ and $A_{3}$. We observe for all the cases, that the error evolves with the speed and the delays for each anchor. But this is not the case in the simulation for $\mathrm{P} 2 \mathrm{P}-\mathrm{B}$. If the duration of one 3-WR transaction is lower than $10 \mathrm{~ms}$, the level of error will not be affected by the speed, since the simulation change the positions of nodes according to the mobility sampling. Considering this issue with more anchors, we can expect to have the same level of ranging error with the anchors performing their transactions during the same interval. Therefore, when designing MAC protocols through simulation, it is necessary to consider the correct sampling of mobility to achieve accurate performance results.

\section{CONClusion}

In this paper, we investigated the impact of speed on the ranging estimation of mobile nodes in a WBAN. We modeled this impact with a theoretical model of the 3 -WR protocol with nodes under mobility. Then, we quantified this effect by using a realistic Yoga scenario and we evaluate the ranging error by simulation with two MAC strategies: P2P-B and A\&B. Moreover, we show that the theoretical error is correlated with the variation of the nodes speed which let us determine the movement direction depending on the sign of the error. Finally, we showed with the theoretical results that the nodes speed (and its associated mobility model) has an impact on the design of MAC strategies. In the future, we will extend this analysis to other scenarios and with a physical channel.

\section{ACKNOWLEDGMENT}

This work has been carried out in the frame of the CORMORAN project, which is funded by the French National Research Agency (ANR) under the contract number ANR-11INFR-010. A part of this research has been realized at the LINCS laboratory. We thank all the involved researchers from ENS/IRISA and M2S Lab for their precious support during the measurement campaign.

\section{REFERENCES}

[1] R. Cavallari, F. Martelli, R. Rosini, C. Buratti, and R. Verdone, "A survey on wireless body area networks: Technologies and design challenges," IEEE Communications Surveys \& Tutorials, vol. 16, no. 3, pp. 1635$1657,2014$.

[2] Z. Xiao, Y. Hei, Q. Yu, and K. Yi, "A survey on impulse-radio UWB localization," Sci. China Inf. Sci., vol. 53, pp. 1322-1335, Jul 2010.

[3] H. Soganci, S. Gezici, and H. Poor, "Accurate positioning in ultrawideband systems," IEEE Wireless Communications, vol. 18, no. 2, pp. 19-27, April 2011.

[4] B. Denis, N. Amiot, B. Uguen, A. Guizar, C. Goursaud, A. Ouni, and C. Chaudet, " Qualitative Analysis of RSSI Behavior in Cooperative Wireless Body Area Networks for Mobility Detection and Navigation Applications,' in 21st IEEE International Conference on Electronics Circuits and Systems, (Marseille, France), Dec. 2014.

[5] "Vicon." http://www.vicon.com/.

[6] A. Guizar, A. Ouni, C. Goursaud, N. Amiot, and J. Gorce, "Impact of MAC scheduling on positioning accuracy for motion capture with UWB body area networks," Proceedings of the 9th International Conference on Body Area Networks, October 2014.

[7] A. Guizar, A. Ouni, C. Goursaud, C. Chaudet, and J. Gorce, "Quantifying the impact of scheduling and mobility on IR-UWB localization in body area networks," in 12th IEEE International Conference on Body Sensor Networks, (MIT, Cambridge, USA), June 2015.

[8] D. Macagnano, G. Destino, F. Esposito, and G. Abreu, "MAC performances for localization and tracking in wireless sensor networks," 2007 4th Workshop on Positioning, Navigation and Communication.

[9] G. Chelius, A. Fraboulet, and E. Ben Hamida, "http://wsnet.gforge.inria.fr/." 